\title{
Sheng Jiang San, a traditional multi-herb formulation, exerts anti-influenza effects in vitro and in vivo via neuraminidase inhibition and immune regulation
}

Tianbo Zhang ${ }^{1}$, Mengjie Xiao ${ }^{2,3}$, Chun-Kwok Wong ${ }^{4,5}$, Ka-Pun Chris Mok ${ }^{6}$ Xin Zhao ${ }^{2,7}$, Huihui Ti ${ }^{2,6^{*}}$ and Pang-Chui Shaw ${ }^{1,5,7^{*}}$ (iD

\begin{abstract}
Background: Sheng Jiang San (SJS), a multi-herb formulation, is used in treating high fever, thirsty and anxiety in ancient China and it is sometimes used to treat seasonal influenza nowadays. However, there is no evidence-based investigation and mechanism research to support the anti-influenza efficacy of SJS. This study aims at evaluating the anti-influenza effect of SJS and investigating its possible mechanism.

Methods: The inhibitory effect of SJS against different influenza virus strains on MDCK cells was examined. Influenza virus infected BALB/c mice were employed to evaluate the efficacy as in vivo model. Mice challenged with A/PR/8/34 (H1N1) were orally administrated $1 \mathrm{~g} / \mathrm{kg} /$ day of SJS for seven days and monitored for 14 days. The survival rate, body weight changes, lung index, lung viral load, histopathologic changes and immune regulation of the mice were measured. The underlying anti-influenza virus mechanism of SJS was studied by a series of biological assays to determine if hemagglutinin, ribonucleoprotein complex or neuraminidase were targets of SJS.

Results: Results showed SJS exerted a broad-spectrum of inhibitory effects on multiple influenza strains in a dose-dependent manner. $\mathrm{C}_{50}$ of SJS against A/WSN/33 (H1N1) was lower than $35 \mu \mathrm{g} / \mathrm{ml}$. SJS also protected 50\% of mice from A/PR/8/34 ( $\mathrm{H} 1 \mathrm{~N} 1)$ infection. The lung index and the lung viral load of SJS treated mice were significantly decreased compared with untreated mice. Meanwhile, SJS targeted on neuraminidase of influenza virus as SJS at $2 \mathrm{mg} / \mathrm{ml}$ inhibited $80 \%$ of neuraminidase enzymatic activity. SJS also significantly down-regulated TNF-a and up-regulated IL-2 of influenza virus induced mice.
\end{abstract}

Conclusions: Thus, SJS is a useful formulation for treating influenza virus infection.

Keywords: Sheng Jiang san, Anti-influenza activity, Neuraminidase inhibition, Immune regulation

\section{Background}

Influenza is a contagious respiratory illness causing seasonal epidemics and occasional pandemics. The death toll of influenza epidemics is between 250,000 to 500,000 . The frequent reassortment of influenza virus may cause high

\footnotetext{
*Correspondence: tihuihui@gzhmu.edu.cn; pcshaw@cuhk.edu.hk

${ }^{2}$ State Key Laboratory of Respiratory Disease, Guangdong Provincial Key Laboratory of Molecular Target \& Clinical Pharmacology, School of Pharmaceutical Sciences and The Fifth Affiliated Hospital, Guangzhou Medical University, Guangzhou 510632, People's Republic of China

'School of Life Sciences, The Chinese University of Hong Kong, Shatin, N.T, Hong Kong, SAR 999077, People's Republic of China

Full list of author information is available at the end of the article
}

mortality and over-burden the healthcare system [1]. For example, the outbreak of $2009 \mathrm{H} 1 \mathrm{~N} 1$ pandemic (swine flu) caused around 185,000 of people death [2]. The most recent 2013 H7N9 is the largest annual epidemics in China also caused significant morbidity and mortality [3].

To date, two classes of anti-influenza drugs are commonly used [4]. One consists of inhibitors of the M2 ion channel, such as amantadine and rimantadine. Treatment with these drugs results in the emergence of resistant strains thus it is not recommended for general use. The other consists of neuraminidase inhibitors, such as oseltamivir, zanamivir, laninamivir and peramivir. In addition,

(c) The Author(s). 2018 Open Access This article is distributed under the terms of the Creative Commons Attribution 4.0 International License (http://creativecommons.org/licenses/by/4.0/), which permits unrestricted use, distribution, and reproduction in any medium, provided you give appropriate credit to the original author(s) and the source, provide a link to the Creative Commons license, and indicate if changes were made. The Creative Commons Public Domain Dedication waiver (http://creativecommons.org/publicdomain/zero/1.0/) applies to the data made available in this article, unless otherwise stated. 
ribavirin and favipiravir (T-705) show anti-viral RNA polymerase effect $[5,6]$. However, resistance against these drugs has already emerged in recent years [7]. These highlight the urgent need for new anti-influenza agents.

Traditional herbal medicine remains an underexplored, yet potentially fruitful basis for antiviral discovery [8]. In ancient China, some Chinese prescriptions were used to treat Wen Bing (Warm Disease), which is considered as influenza in modern time, with influenza-like symptoms, such as high fever, thirsty and anxiety [9]. To date, these prescriptions are still used in clinics by traditional Chinese medical practitioners. Also, in South China, multi-herb drink or "cooling herbal tea" is a convenient folk treatment against normal cold or mild influenza [10]. However, the efficacy of most of these products has not been vigorously tested. There is no conclusive experimental evidence to support the clinical efficacies of these prescriptions in treating influenza. Nevertheless, researchers have begun to evaluate the therapeutic values and underlying mechanism of selected prescriptions, including Chinese patent drugs and traditional Chinese prescriptions [11-13]. For example, Lianhua Qingwen capsule [14] was shown to have broad-spectrum efficacy on a number of influenza virus strains, through regulating the immune responses after virus infection. Kang Bing Du oral liquid [15] was found to reduce the susceptibility to influenza virus via mitochondrial antiviral signaling.

Sheng Jiang San (SJS) is a famous Chinese prescription that was originally recorded in a Traditional Chinese Medicine Classic Shanghan Wenyi Tiaobian of Qing Dynasty. SJS is composed of Rhei Radix et Rhizoma, Bombyx Batryticatus, Cicadae Periostracum and Curcumae Longae Rhizoma in a ratio of 4:2:1:3 $(w / w / w / w)$. It has been prescribed in treating "Warm Disease". In modern time, traditional Chinese medical practitioners use it to treat seasonal influenza. However, there is no proper statistics on its clinical efficacy and revelation of the anti-influenza virus mechanism. Our preliminary test showed that it could indeed inhibit influenza A/WSN/33 (H1N1) in cell culture. As a contribution to increase the clinical value and the modernization of Chinese medicine, we set forth to examine the influenza inhibitory effect of SJS.

Currently, influenza virus infected mouse model is frequently used to test the in vivo influenza therapeutic efficacy of a drug [11-16]. In this study, except examining the inhibitory effect of SJS against different influenza virus strains on Madin-Darby canine kidney (MDCK) cells, we also used influenza virus infected BALB/c mice as an in vivo model to investigate the therapeutic action of SJS. The underlying anti-influenza virus mechanisms were studied by a battery of biological assays, which include viral absorption and release, and the function of viral polymerase complex.

\section{Methods \\ Reagents}

Rhei Radix et Rhizoma, Bombyx Batryticatus, Cicadae Periostracum and Curcumae Longae Rhizoma were purchased from Zisun Chinese Pharmaceutical Co., Ltd. (Guangzhou, China). Standard compounds of rhein, chrysophanol, emodin, aloe emodin and curcumin were purchased from Chengdu Pufeide Biotechnology Co., Ltd. (Chengdu, China). Oseltamivir was purchased from Yichang Changjiang Pharcaceutical Co., Ltd. (Wuhan, China). Minimum essential medium (MEM), Dulbecco's modified eagle medium (DMEM) and fetal bovine serum (FBS) were purchased from Life Technologies (Gibco, NY, USA). Neuraminidase inhibitors screen kit (no. P0309) was purchased from Beyotime Institute of Biotechnology Co., Ltd. (Shanghai, China). Chicken erythrocytes were purchased from Lampire Biological Laboratories (PA, USA). Tolylsulfonyl phenylalanyl chloromethyl ketone (TPCK) treated-trypsin was purchased from Sigma-Aldrich (St. Louis, MO, USA). Mouse TNF- $\alpha$, IFN- $\alpha$ and IL-2 Enzyme-linked immunosorbent assay (Elisa) kit were purchased from Invitrogen (Carlsbad, CA, USA). Water used in this study was purified by a Milli-Q system (Millipore, MA, USA). All culture plates were obtained from Greiner (Cellstar, Germany).

\section{Preparation of SJS extract}

The identities of Rhei Radix et Rhizoma, Bombyx Batryticatus, Cicadae Periostracum and Curcumae Longae Rhizoma were confirmed by an expert at the Institute of Chinese Medicine, The Chinese University of Hong Kong, by referring to their organoleptic characteristics. The voucher specimens were kept at Li Dak Sum Yip Yio Chin R \& D Centre for Chinese Medicine, The Chinese University of Hong Kong. The aqueous extract of SJS was prepared by boiling the herbs at 4:2:1:3. The four ingredients in proportion were boiled twice with deionized water for $1 \mathrm{~h}$ each time. The aqueous extract was filtered and concentrated by a rotary evaporator under vacuum in a $60{ }^{\circ} \mathrm{C}$ water bath. Then the concentrated extract was lyophilized into powder under vacuum of $105 \times 10^{-3}$ mbar and $-40{ }^{\circ} \mathrm{C}$. The freeze-dried powder was dissolved in culture medium or water before used.

Quality control is important in Chinese prescription, as the consistency will affect the repeatability of experiments and clinical efficacy. In light of this, a large amount of freeze-dried powder of SJS was prepared only once for studies to avoid composition differences between different batches of herbs. SJS powder was analyzed by highperformance liquid chromatography (Additional file 1) 
and the chemical profile is shown in Additional file 2: Figure S1. By comparing with reference compounds, rhein, chrysophanol, emodin, aloe emodin and curcumin were found.

\section{Cells, viruses and animals}

MDCK cells and human embryonic kidney $293 \mathrm{~T}$ (293 T) cells were obtained from American Type Culture Collection and routinely cultured in MEM and DMEM, respectively, supplemented with 10\% FBS and incubated at $37{ }^{\circ} \mathrm{C}$ with $5 \% \mathrm{CO}_{2}$. Influenza A/WSN/33 (H1N1) (WSN), A/PR/8/34 (H1N1) (PR8), A/GZ/GIRD07/09 (H1N1), A/HK/8/68 (H3N2), A/Aichi/2/1968 (H3N2), A/ HK/Y280/97 (H9N2), A/China/24/96 (H7N3), B/Lee/1940 (Flu B) were provided by Dr. Zifeng Yang (Guangzhou Institute of Respiratory Disease, China). All in vitro tests were performed in class II biosafety cabinet.

Specific-pathogen-free Balb/c mice weighing 14-16 g were used in this study. Mice were obtained from Guangdong Medical Laboratory Animal Center (Guangzhou, China). The animal experiments were carried out according to the Guidelines of Guangdong Regulation for the Administration of Laboratory Animals. The mice were kept in biosafety level 3 housing and provided with standard laboratory diet and water ad libitum.

\section{Cytotoxicity assay}

Cytotoxic effect of SJS was assessed by 3-(4,5-dimethylthiazol-2-yl)-2,5- diphenyltetrazolium bromide (MTT) assay. MDCK cells $\left(2 \times 10^{5}\right)$ were seeded on a 96-well culture plate in MEM with 10\% FBS. After overnight culture, cells were treated with different concentration of SJS in MEM. After $24 \mathrm{~h}$ incubation at $37{ }^{\circ} \mathrm{C}$, MTT $(5 \mathrm{mg} / \mathrm{ml})$ in phosphate buffered saline (PBS) was freshly prepared, $10 \mu \mathrm{l}$ of MTT solution was added to each well and the plates were incubated at $37{ }^{\circ} \mathrm{C}$ for $4 \mathrm{~h}$. The medium was then removed and formazan crystal was dissolved in dimethyl sulfoxide (DMSO) $(100 \mu \mathrm{l} /$ well). Then the absorbance at $570 \mathrm{~nm}$ was read by a CLARIOstar multi-mode microplate reader (BMG Labtech, Germany). The $50 \%$ toxic concentration $\left(\mathrm{TC}_{50}\right)$ was calculated as the concentration required to decrease $50 \%$ of cell viability.

\section{Cytopathic effect inhibition (CPE) assay}

$80 \%$ confluent MDCK cells in a 96-well plate were infected with $0.01 \mathrm{MOI}$ of influenza virus for $1 \mathrm{~h}$ at $37^{\circ} \mathrm{C}$. Afterwards, the viral inoculum was removed, and cells were washed twice with PBS. $100 \mu \mathrm{l}$ SJS at different concentration in serum-free MEM with $1 \mu \mathrm{g} / \mathrm{ml}$ TPCK treated-trypsin (TPCK treated-trypsin was absent when MDCK cells were infected by WSN virus) was added to the cells. After incubating at $37{ }^{\circ} \mathrm{C}$ for $48 \mathrm{~h}, 10 \mu \mathrm{l}$ of $5 \mathrm{mg} / \mathrm{ml}$ fresh MTT solution in PBS was added to each well and the plates were incubated at $37^{\circ} \mathrm{C}$ for $4 \mathrm{~h}$. The medium was then removed and formazan crystal was dissolved in DMSO (100 $\mu \mathrm{l} /$ well). Absorbance at $570 \mathrm{~nm}$ was read by a CLARIOstar multi-mode microplate reader (BMG Labtech, Germany). The concentration that inhibited $50 \%$ of virus-induced cytopathic effect was determined as $\mathrm{IC}_{50}$.

\section{Plaque reduction assay}

Confluent MDCK cells were seeded in 6-well plates in MEM with $10 \%$ FBS. Cells were infected with around 200 pfu per well of different viral strains for $1 \mathrm{~h}$ at $37^{\circ} \mathrm{C}$. The inoculum was aspirated to remove unbound viral particles, followed by washing with PBS. The MDCK monolayer was then overlaid with $1 \%$ low melting agarose (Cambrex) in MEM which contained different concentration of SJS and $1 \mu \mathrm{g} / \mathrm{ml}$ of TPCK treated-trypsin (TPCK treated-trypsin was absent when MDCK cells were infected by WSN virus). After incubating for $72 \mathrm{~h}$ at $37{ }^{\circ} \mathrm{C}$, the agarose was removed and the cell monolayers were stained with staining solution $(0.25 \%$ coomassie blue, $10 \%$ acetic acid, 50\% methanol). The number of plaques was counted and the percentage of plaque inhibition relative to the control (no drug treatment) was calculated.

\section{Multicycle growth assay}

$80 \%$ confluent MDCK cells were seeded in a 24-well plate. After infecting with $0.001 \mathrm{MOI}$ of WSN for $1 \mathrm{~h}$ at $37{ }^{\circ} \mathrm{C}$, the inoculum was removed and $500 \mu \mathrm{l}$ of SJS $(500 \mu \mathrm{g} / \mathrm{ml}, 125 \mu \mathrm{g} / \mathrm{ml}, \quad 60 \mu \mathrm{g} / \mathrm{ml}$ ) or oseltamivir $(100 \mu \mathrm{M})$ in MEM, or $500 \mu \mathrm{l}$ MEM only were added to the cells and incubated at $37^{\circ} \mathrm{C}$. The supernatants were then collected at 12, 24, 48 and $72 \mathrm{~h}$ post infection. The virus titers were determined by plaque assay as described previously [17].

\section{Hemagglutination inhibition assay}

Twofold serial dilution of SJS was prepared in $25 \mu \mathrm{l}$ of PBS in a 96-well U-bottom plate. WSN in $25 \mu \mathrm{l}$ of PBS (4 HA units) was added to each dilution and mixed well, and the plate was incubated for $30 \mathrm{~min}$ at room temperature. Then, $50 \mu \mathrm{l}$ of chicken erythrocytes in PBS $(0.05 \% v / \mathrm{v})$ was added to each well and mixed thoroughly. The reaction was observed after incubating the plates at room temperature for another $30 \mathrm{~min}$. Pentagalloyglucose (PGG) was used as a positive control [18] while oseltamivir was a negative control [19].

\section{Ribonucleoprotein (RNP) reconstitution assay}

$2 \times 10^{6}$ of $293 \mathrm{~T}$ cells were seeded on a $6 \mathrm{~cm}$ dish and incubated overnight in DMEM with 10\% FBS. Plasmids pcDNA3a-PB1, pcDNA3a-PB2, pcDNA3a-PA, pcDNA3aNP, pPOL-NS-Luci (kindly provided by Dr. Ervin Fodor, 
University of Oxford, UK) were transfected to $293 \mathrm{~T}$ cells with Lipofectamine 2000 (Invitrogen, CA, USA) to reconstitute the RNP complex. The RNP complex consisted of WSN polymerase proteins $\mathrm{PA}, \mathrm{PB} 1$ and $\mathrm{PB} 2, \mathrm{NP}$ and a luciferase reporter gene. Plasmid pEGFP was also cotransfected to $293 \mathrm{~T}$ cells as an internal control to normalize the transfection efficiency. After $6 \mathrm{~h}$ of transfection, transfected cells were trypsinized and aliquoted into a 96-well plate. SJS at different concentration dissolved in DMEM was added into each well. After incubating for $24 \mathrm{~h}$ at $37^{\circ} \mathrm{C}$, the cell lysates were harvested and the luciferase activity was assayed with a luciferase reporter assay system kit (Promega, No. E1910). The luminescence was read by a CLARIOstar multi-mode microplate reader (BMG Labtech, Germany).

\section{Neuraminidase (NA) inhibition assay}

A neuraminidase inhibitors screen kit was employed to evaluate the inhibition of SJS on the NA enzymatic activity. The assay followed the instruction manual. $70 \mu \mathrm{l}$ of reaction buffer, $10 \mu \mathrm{l}$ of NA and $10 \mu \mathrm{l}$ of SJS at different concentration were well mixed in a black 96-well microplate. After incubating at $37{ }^{\circ} \mathrm{C}$ for $2 \mathrm{~min}, 10 \mu \mathrm{l}$ of substrate was added into each well, mixed thoroughly and incubated for $1 \mathrm{~h}$. Fluorescence was measured with a CLARIOstar multi-mode microplate reader (BMG Labtech, Germany) at an excitation wavelength of $322 \mathrm{~nm}$ and an emission wavelength of $450 \mathrm{~nm}$. Oseltamivir acid [19] was used as a positive control. The NA activity inhibitory percentage was calculated as follow:

$$
\begin{aligned}
& \text { NA inhibition }(\%)=\left(\mathrm{F}_{\text {control }}-\mathrm{F}_{\mathrm{SJS}}\right) /\left(\mathrm{F}_{\text {control }}-\mathrm{F}_{\text {blank }}\right) \\
& \times 100 \%(\mathrm{~F}: \text { Fluorescence intensity }) .
\end{aligned}
$$

\section{Anti-influenza virus test in mouse model}

Mice were randomly divided into vehicle group, SJS group, oseltamivir group and untreated group, with 16 mice in each group. Except the vehicle group, other groups were anaesthetized with ethyl ether and inoculated intranasally with $3 \mathrm{LD}_{50}(50 \%$ lethal dose) of mouse-adapted PR8 virus in a volume of $50 \mu \mathrm{l}$. At $4 \mathrm{~h}$ after inoculation, SJS group and oseltamivir group were treated by gavage feeding with SJS solution (dissolved in water at a dose of $1 \mathrm{~g} / \mathrm{kg} /$ day) or oseltamivir solution (dissolved in water at a dose of $90 \mathrm{mg} / \mathrm{kg} /$ day) in a volume of $200 \mu \mathrm{l}$, respectively. Then these two groups were administrated orally once daily for seven consecutive days. Untreated group and vehicle group were fed with water. Parameters of mice such as mortality, body weight and general conditions were monitered for consecutive 14 days.

Three mice from each group were randomly selected and sacrificed on the fourth day post-inoculation for lung index calculation, lung viral load titer and lung cytokine expression analysis. Another three mice from each group were also sacrificed on the sixth day postinoculation for histopathologic observation. The sacrificed mice were euthanized by cervical dislocation after fully anaesthetization by inhaling diethyl ether. The remaining ten mice in each group were monitored continuously for 14 consecutive days to study their mortality and body weight changes.

\section{Lung index}

Four days after virus infection, mice were weighed and their lung tissues were extracted and washed with PBS, dried by gauze and then weighed. Lung index was calculated as follow:

Lung index $=$ lung weight/body weight $\times 100 \%$.

\section{Lung viral load titer}

After weighing the lung tissues, they were homogenized in MEM by a refiner (Qiagen, TissueRuptor) and centrifuged at $12000 \mathrm{rpm}$ for $5 \mathrm{~min}$ at $4{ }^{\circ} \mathrm{C}$. The lung homogenates were aliquoted and stored at $-80{ }^{\circ} \mathrm{C}$. The virus titer of these homogenates were determined by plaque assay [17] on MDCK cells.

\section{Lung cytokine expression analysis}

Part of the lung homogenates was used to perform lung cytokine expression analysis with mouse cytokines Elisa kits of TNF- $\alpha$, IFN- $\alpha$ and IL-2 (Invitrogen). The content of TNF- $\alpha$, IFN- $\alpha$ and IL- 2 were assessed according to the manufacturer's protocol. Absorbance at $450 \mathrm{~nm}$ was read by a spectro-photometer (Thermo Scentific).

\section{Histopathologic observation}

Six days after virus infection, lung tissues were extracted from three randomly sacrificed mice from each group. The lungs were immersed in $10 \%$ formaldehyde solution immediately and embedded in paraffin. Then the lung tissue was cut into $4 \mu \mathrm{m}$-thick sections. Tissue sections were stained with hematoxylin and eosin for observating the histopathologic changes under microscope.

\section{Statistics}

All statistical analyses were conducted with Graphpad Prism 6.0 (Graphpad, San Diego, CA, USA) and data were presented as mean \pm SD. One-way ANOVA was used for multiple-group comparison. Differences were considered statistically significant when $p<0.05\left({ }^{*} p<0.05,{ }^{* *} p<0.01\right.$, **** $p<0.001)$. 


\section{Results}

Anti-influenza activity of SJS against multiple virus strains To determine the inhibitory activity of SJS against the cytopathic effect induced by different viral strains, CPE was performed. Plaque reduction assay was also performed to confirm the antiviral efficacy of SJS on A/ WSN/33 (H1N1), A/PR/8/34 (H1N1), A/GZ/GIRD07/09 (H1N1), A/Aichi/2/1968 (H3N2), A/HK/Y280/97 (H9N2) and A/China/24/96 (H7N3). B/Lee/1940 (Flu B) was only tested by $\mathrm{CPE}$ assay. The $\mathrm{IC}_{50}$ on each strain was calculated based on the results of CPE assay and is shown in Table 1 . SJS had a $\mathrm{TC}_{50}>2 \mathrm{mg} / \mathrm{ml}$ measured by cytotoxicity assay. The selective index (SI) of each strain was also calculated and shown in Table 1 . The $\mathrm{IC}_{50}$ was ranging from 34.7 to $750.8 \mu \mathrm{g} / \mathrm{ml}$, and SI was ranging from 2.7 to 57.7. SJS showed the best inhibitory effect on WSN virus $\left(\mathrm{IC}_{50}=34.7\right.$ and $\left.\mathrm{SI}=57.7\right)$. According to the results of plaque reduction assay, SJS inhibited the virus strains in a dose-dependent manner. It inhibited the growth of all the seven viruses (200 pfu per well) to $100 \%$ at less than $1 \mathrm{mg} / \mathrm{ml}$ (Fig. 1a).

To evaluate the influence of SJS on viral progeny, MDCK cells were treated with SJS at $125 \mu \mathrm{g} / \mathrm{ml}$ and $500 \mu \mathrm{g} / \mathrm{ml}$ after infected with WSN virus $(\mathrm{MOI}=0.001)$. The supernatant with replicated virus was collected at $12,24,48$ and $72 \mathrm{~h}$, and the viral titer at each time point was measured by plaque assay. As shown in Fig. 1b, SJS severely suppressed the WSN viral multicycle growth at $500 \mu \mathrm{g} / \mathrm{ml}$. On the other hand, oseltamivir as a positive control inhibited viral production at $200 \mu \mathrm{M}$ at $72 \mathrm{~h}$. This indicated that SJS had a pronounced effect on suppressing the WSN growth.

\section{SJS did not act on HA and RNP complex}

To determine whether SJS could inhibit hemagglutinin of virus particles for binding to cell surface receptors, hemagglutination inhibition assay was performed. Influenza virus can agglutinate erythrocytes by means of hemagglutination, then erythrocytes become cross-linked and form lattice. In this assay, chicken erythrocytes

Table 1 Antiviral activity of SJS against different influenza virus strain

\begin{tabular}{lll}
\hline Viral strains & $\mathrm{IC}_{50}{ }^{\mathrm{a}}(\mu \mathrm{g} / \mathrm{ml})$ & $\mathrm{SI}^{\mathrm{b}}(>)$ \\
\hline A/PR/8/34 (H1N1) & 198.47 & 10.08 \\
A/WSN/33 (H1N1) & 34.66 & 57.70 \\
A/GZ/GIRD07/09 (H1N1) & 78.56 & 25.46 \\
A/China/24/96 (H7N3) & 371.13 & 5.39 \\
A/HK/Y280/97 (H9N2) & 353.55 & 5.66 \\
A/Aichi/2/1968 (H3N2) & 360.73 & 5.54 \\
B/Lee/1940 (Flu B) & 750.79 & 2.66 \\
\hline
\end{tabular}

${ }^{a}$ Mean of the results from three independent experiments

${ }^{\mathrm{b}}$ The $\mathrm{SI}$ (selectivity index) was calculated as the ratio of $\mathrm{TC}_{50}$ to $\mathrm{IC}_{50}$

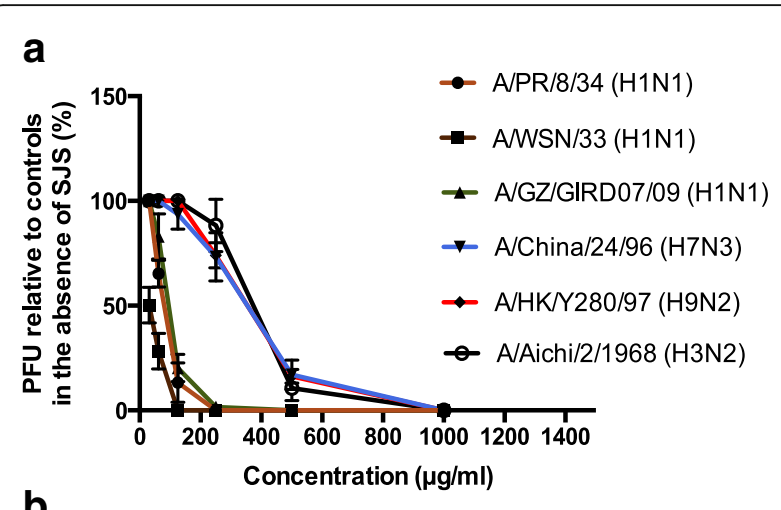

b

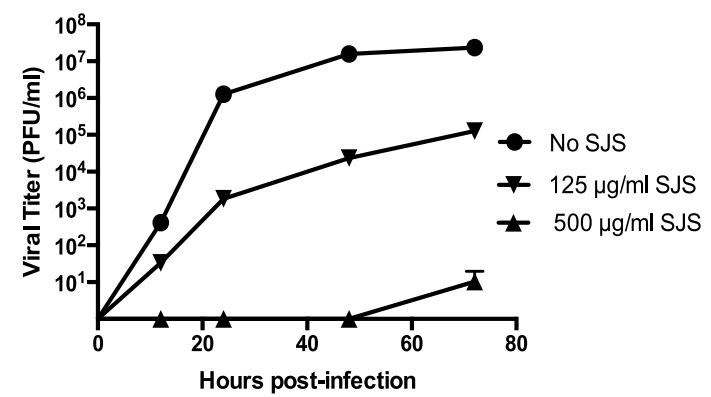

Fig. 1 Antiviral activity of SJS in cell culture (a) SJS inhibited seven influenza viruses on MDCK cells in plaque reduction assay. Oseltamivir at $100 \mu \mathrm{M}$ stopped viral growth but data was omitted for clarity. b Antiviral effect of SJS in multicycle growth assay. MDCK cells were infected with WSN at MOI $=0.001$ in the presence or absence of SJS and the viral progeny at $12,24,48$ and $72 \mathrm{~h}$ were determined by plaque assay. SJS at $1 \mathrm{mg} / \mathrm{ml}$ and oseltamivir at $100 \mu \mathrm{M}$ inhibited viral growth till $72 \mathrm{~h}$. The experiments were carried out in duplicate and repeated three times for confirmation

showed a lattice appearance when treated with SJS at 63$500 \mu \mathrm{g} / \mathrm{ml}$ (in two-fold serial dilution) in the presence of WSN virus (4 HA units) (Fig. 2a). Chicken erythrocytes treated with the positive control PGG at 6-50 $\mu \mathrm{M}$ and WSN virus showed red spot like appearance, indicating the inhibition of hamagglutination. Oseltamivir, on the other hand, had no effect on HA. When WSN was absent, SJS and PGG treatment also showed a red spot appearance, which indicated they had no influence to chicken erythrocytes.

Influenza RNP reconstitution assay was performed to evaluate if SJS inhibited the viral RNP activity. SJS were added to 293 T cells transfected with WSN minigenomes to 250,500 and $1000 \mu \mathrm{g} / \mathrm{ml}$. Luciferase activity was measured after $24 \mathrm{~h}$ post-transfection. Nucleozin [16], an NP inhibitor, was used as a positive control in the assay. As shown in Fig. 2b, the luciferase activity in the presence of SJS had no obvious differences with the untreated control, while nucleozin inhibited the luciferase activity significantly.

Thus, SJS did not interfere with the absorption of WSN towards the target cells or viral polymerase activity. 


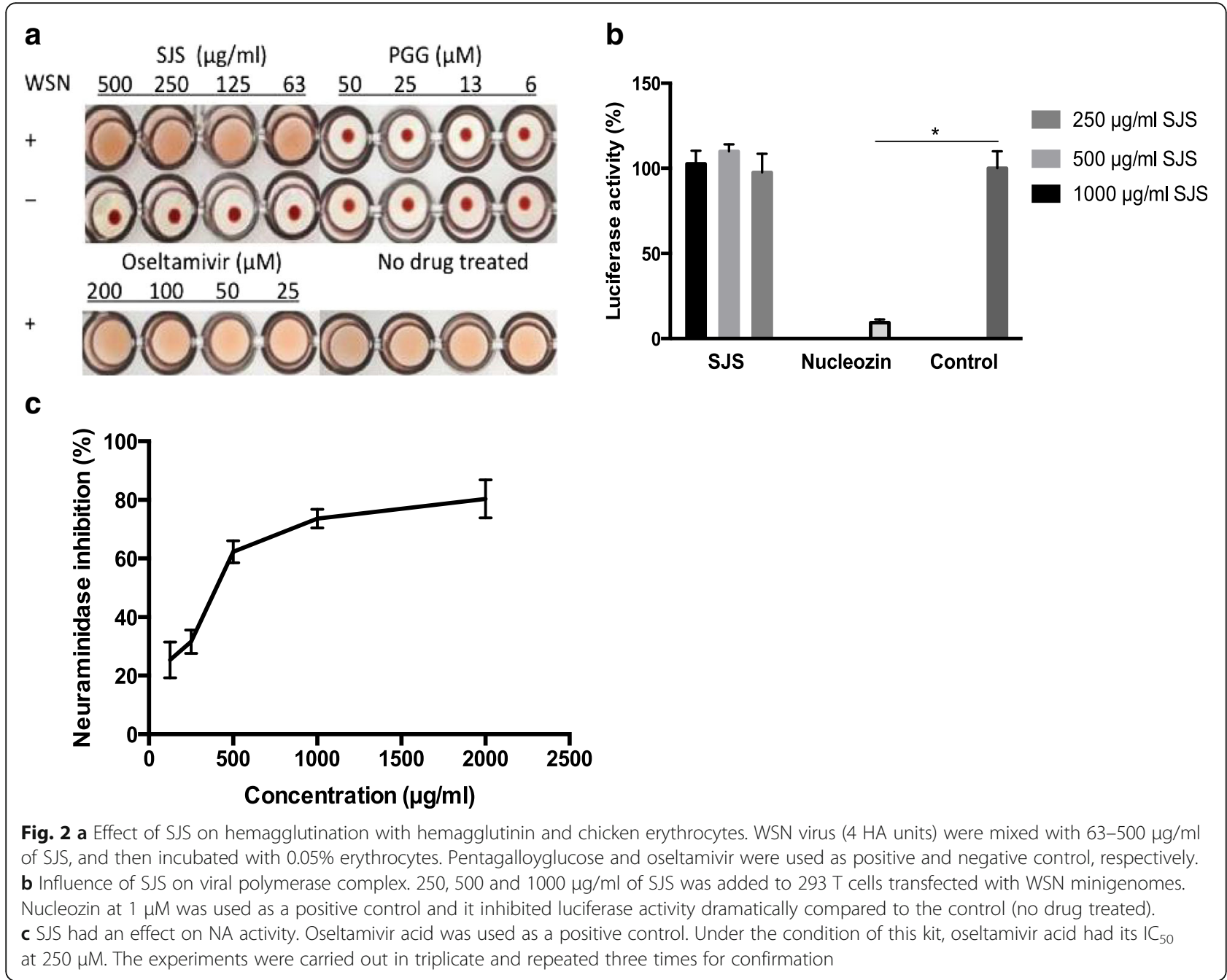

\section{SJS inhibited NA activity}

To explore if SJS affected NA enzymatic activity, NA inhibitory assay was conducted using a commercial neuraminidase inhibitors screen kit. Two-fold serial dilutions from $125 \mu \mathrm{g} / \mathrm{ml}$ to $2000 \mu \mathrm{g} / \mathrm{ml}$ of SJS extract were tested. NA activity was measured by fluorescence of 4methylumbelliferone, which was the product of substrate (4-Methylumbelliferyl)-a-D-N-acetylneuraminic acid sodium salt hydrate catalyzed by the enzymatic activity of NA. Results showed that at $2 \mathrm{mg} / \mathrm{ml}$ of SJS, NA activity was inhibited up to $80 \%$ (Fig. 2(c)). Oseltamivir acid was used as a positive control and it inhibited 50\% NA activity at $250 \mu \mathrm{M}$.

\section{SJS improved survival rate of PR8-infected mice}

To evaluate the in vivo antiviral efficacy of SJS, groups of mice were inoculated with $3 \mathrm{LD}_{50}$ of mouse lung-adapted viral strain PR8 and orally administrated SJS for seven consecutive days, while vehicle group and untreated group were administrated with water instead. Vehicle control group showed normal appearance and behaviors during the 14-day observation. Mice in the untreated group all died before the 8th day post-inoculation of virus. They also showed inactive, ruffled fur and respiratory distress signs. Mice orally administrated with SJS daily had their life span prolonged. This group showed mortality on day 9 post-inoculation and up to $50 \%$ mice survived after 14 days (Fig. 3a). Besides, the average body weight of SJS group rebound on day 9 post-inoculation, similar to the positive control (oseltamivir) group (Fig. 3b). These results suggested that SJS at $1 \mathrm{~g} / \mathrm{kg} /$ day had significant protective effect on mice infected with PR8 virus.

\section{SJS reduced lung index, lung viral load and alleviated} lung histopathologic changes of PR8 virus infected mice. Three mice from each group were euthanized on day 4 post-inoculation and their lungs were extracted for lung index measurement and lung viral load titer. Compared with the untreated group, SJS at $1 \mathrm{~g} / \mathrm{kg} /$ day significantly 


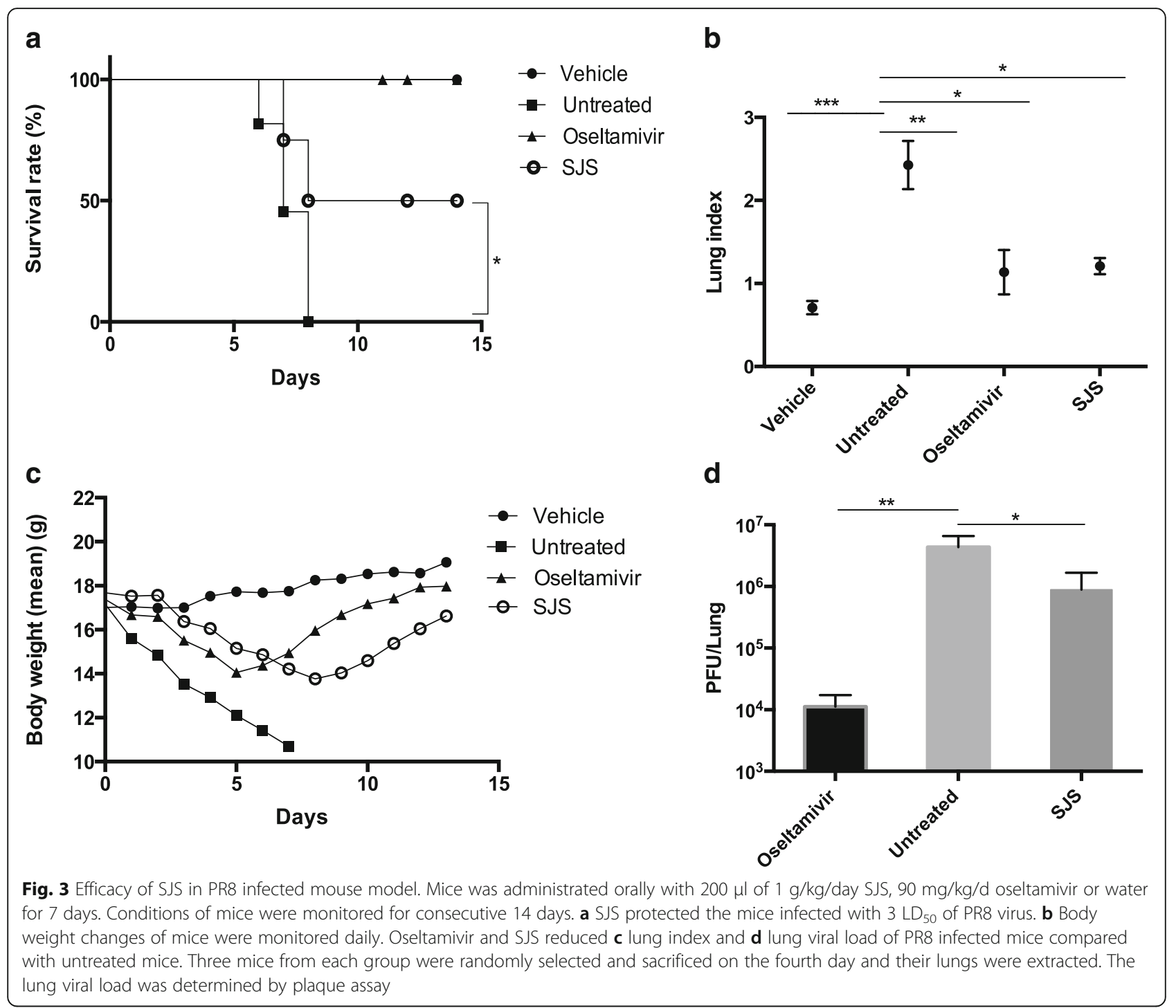

decreased the mice lung index (Fig. 3c) and inhibited the lung viral load (Fig. 3d).

Another three mice were also euthanized on the sixth day post-inoculation for observating the histopathologic changes. As shown in Fig. 4, untreated group showed pronounced lung inflammation, characterized by interstitial expansion, edema and inflammatory cell infiltration around small vessels. Inflammation cells could be observed in alveolar lumen. For SJS group, the histopathology was alleviated and mild lesions were observed. Less inflammatory cells were exuded and infiltrated around vessels and interstitial space. The results of lung index and

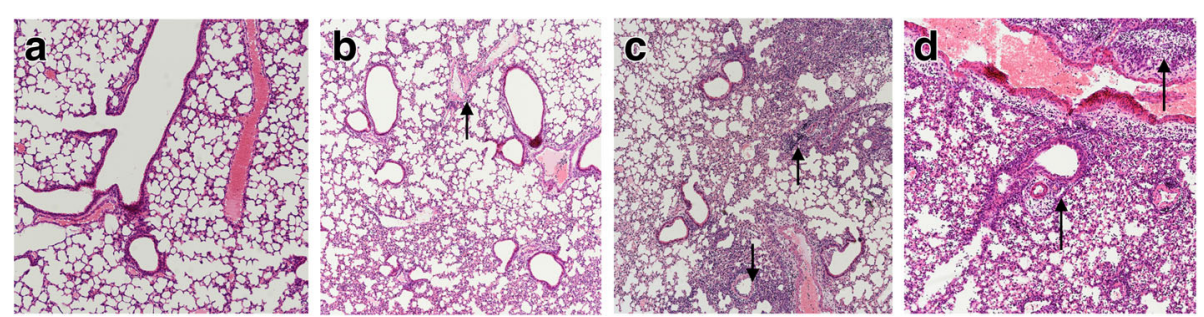

Fig. 4 Lung hisotopathologic changes of mice were examined on the sixth day. Representative histologic sections of sacrificed mice from a Vehicle group b Oseltamivir group c SJS group $\mathbf{d}$ Untreated group were visualized using hematoxylin and eosin staining. The black arrows in B-D highlight the area of inflammatory cell infiltration in submucosal layer of airway epithelium and perivascular region. (Magnification: 100X) 
lung viral load following SJS treatment indicated that SJS treatment alleviated lung pathology and lesion of PR8 infected mice.

\section{Effect of SJS on lung cytokine expression}

To determine the inflammatory markers after SJS treatment, part of the lung homogenates collected on day 4 post-inoculation was used for Elisa assay on the level of cytokine TNF- $\alpha$, IFN- $\alpha$ and IL-2 (Fig. 5). For the former two markers, their expression in infected group increased significantly compared with the vehicle group. Oseltamivir and SJS treatment both decreased the expression level of these two cytokines after PR8 infection, though IFN- $\alpha$ was only slightly reduced. Cytokine IL-2 in mice lung decreased after PR8 infection, oseltamivir and SJS displayed the same tendency that they increased the level of IL-2. In this Elisa analysis, SJS also showed better regulatory activity on TNF- $\alpha$ and IFN- $\alpha$ than oseltamivir group. These results showed that SJS could reduce the inflammatory responses in mice.

\section{Discussion}

SJS is a classical Chinese formulation for treating influenza virus infection. However, there has been no systematic study to substantiate the anti-influenza efficacy. The mechanism of SJS action also remained unclear.

We first found that SIS could inhibit flu A and B strains on MDCK cell line at less than $750 \mu \mathrm{g} / \mathrm{ml}$, showing that SJS has a broad-spectrum inhibitory activity to influenza viruses. We also showed that SJS could protect mice from PR8 infection. Compared with the untreated group, SJS could significantly improve the survival rate and prolong the average survival day, also help the body weight of the mice to rebound. SJS treated mice not only had a lower lung viral load but also had reduced lung index and alleviated histopathology, suggesting that SJS might work on the virus directly and SJS could also decrease the lung injury induced by PR8 infection. Compared with oseltamivir, SJS is less effective in protecting

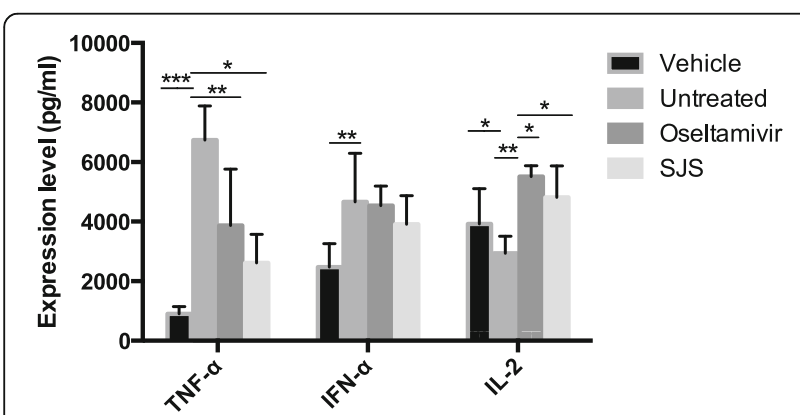

Fig. 5 Influence of SJS on cytokine expression in mouse lung infected with PR8 virus. The cytokine expression level of TNF-a, IFN-a and IL-2 was analyzed using Elisa. The experiments were carried out in triplicate and repeated three times for confirmation
PR8 infected mice. However, considering that the formulation containing many phytochemicals, with each in a minute quantity, there is a high chance to have some potent fractions or phytochemicals in the extract.

Previous reports pointed out that over-expression of TNF- $\alpha$ and IFN- $\alpha$ induced by influenza virus is a cause of lung inflammation and can in turn result in tissue injury [20, 21]. In contrast, IL-2 decreases after infected by influenza virus and lower expression of IL-2 causes dysfunction of immune system [11]. In this study, the expression level of three cytokines (TNF- $\alpha$, IFN- $\alpha$, IL-2) after PR8 virus infection was examined. Our data showed SJS was beneficial to PR8 infected mice, as SJS could down-regulate TNF- $\alpha$ and up-regulate IL-2. The expression level of IFN- $\alpha$ under treatment of SJS also showed a down-regulatory trend, although there was no statistically significant difference with the untreated group. Meanwhile, compared with the treatment of oseltamivir, SJS showed a stronger regulation on the expression of these cytokines (Fig. 5). Thus, besides inhibiting virus replication directly through targeting on NA, SJS might also protect the mice through regulating the expression of cytokines. We are in the process of carrying out bioassay-guided fractionation to find the pure phytochemicals that inhibit NA and regulate the concerned cytokines.

As influenza infection is a common disease, there are many cold and flu herbal remedies in the market, such as Chinese patent drugs, traditional Chinese prescriptions, or even cooling herbal tea. However, their efficacy in treating influenza is not well investigated. Our research has provided an example on the evidence-based research on an anti-influenza formulation, which involves virus inhibitory studies in cell culture, animal model and mechanism elucidation. The work may be extended to other traditional or folk medicine, which will strengthen the confidence on their clinical uses and for downstream development of these formulations.

\section{Conclusions}

In this study, a Chinese prescription SJS was found to inhibit a number of influenza virus strains and act against influenza virus PR8 in mice. SIS exhibited anti-influenza activity through inhibiting NA activity and regulating cytokine expression. Our work has substantiated that SJS is an effective anti-influenza formulation, which may be further developed by the pharmaceutical industry.

\section{Additional files}

Additional file 1: Method of High-performance liquid chromatography (HPLC) analysis of SJS. HPLC method was used to analyze the chemical profile of SJS. The HPLC condition is described in this additional file and the profile is shown in Additional file 2: Figure S1. By comparing with 
reference compounds, rhein, chrysophanol, emodin, aloe emodin and curcumin were found. (DOCX $13 \mathrm{~kb}$ )

Additional file 2: Figure S1. HPLC analysis of SJS (a) HPLC profile of SJS (b) Some constituents were denoted by standard compounds. (PPTX $128 \mathrm{~kb})$

\section{Abbreviations}

CPE: Cytopathic effect inhibition; DMEM: Dulbecco's Modified Eagle Medium; DMSO: Dimethyl sulfoxide; Elisa: Enzyme-linked immunosorbent assay; FBS: Fetal bovine serum; HA: Hemagglutinin; $I_{50}$ : $50 \%$ inhibitory concentration; IFN: Interferon; IL: Interleukin; $\mathrm{LD}_{50}$ : 50\% lethal dose; MDCK: Madin-Darby canine kidney; MEM: Minimum essential medium; MOI: Multiplicity of infection; MTT: 3-(4,5-dimethylthiazol-2-yl)-2,5diphenyltetrazolium bromide; NA: Neuraminidase; NP: Nucleoprotein; PA: Polymerase acidic protein; PB1: Polymerase basic protein 1;

PB2: Polymerase basic protein 2; PBS: Phosphate buffered saline; Pfu: Plaque forming unit; PGG: Pentagalloyglucose; PR8: A/PR/8/34 (H1N1);

RNP: Ribonucleoprotein; SI: Selective index; SJS: Sheng Jiang San; $\mathrm{TC}_{50}$ : 50\% toxic concentration; TNF: Tumor necrosis factor; TPCK: Tolylsulfonyl phenylalanyl chloromethyl ketone; WSN: ANSN/33 (H1N1)

\section{Acknowledgements}

The authors would like to extend their sincere appreciation to Dr. Zifeng Yang (Guangzhou Institute of Respiratory Disease, China) and Dr. Ervin Fodor (University of Oxford, UK) for providing viruses and plasmids respectively.

\section{Ethics approval and consent to participant}

All animal research was carried out according to the Guidelines of Guangdong Regulation for the Administration of Laboratory Animals and approved by Guangdong Provincial Department of Science and Technology.

\section{Funding}

This work was supported in part by a Theme-based grant (Project No. T11705/14 N) of the Research Grants Council of Hong Kong HKSAR and a grant from the National Natural Science Foundation of China (no. 31401662) and Science research project of the Guangdong Province (no. 2016A050503047).

\section{Availability of data and materials}

All data generated or analyzed during this study are included in this article and its additional files.

\section{Authors' contributions}

TBZ, HHT, PCS co-conceived the study and designed the experiments. MJX, TBZ, XZ and HHT conducted the cell biology tests. MJX and HHT performed the animal experiments. CKW, KPM and PCS carried on the mechanisms investigation and data analysis. TBZ and PCS contributed to draft the manuscript. All authors read and approved the final manuscript.

\section{Competing interests}

The authors declare that they have no competing interests.

\section{Publisher's Note}

Springer Nature remains neutral with regard to jurisdictional claims in published maps and institutional affiliations.

\section{Author details}

'School of Life Sciences, The Chinese University of Hong Kong, Shatin, N.T, Hong Kong, SAR 999077, People's Republic of China. ${ }^{2}$ State Key Laboratory of Respiratory Disease, Guangdong Provincial Key Laboratory of Molecular Target \& Clinical Pharmacology, School of Pharmaceutical Sciences and The Fifth Affiliated Hospital, Guangzhou Medical University, Guangzhou 510632, People's Republic of China. ${ }^{3}$ Guangzhou Institutes of Biomedicine and Health, Chinese Academy of Science, Guangzhou 510632, People's Republic of China. ${ }^{4}$ Department of Chemical Pathology, The Chinese University of Hong Kong, Prince of Wales Hospital, Shatin, N.T, Hong Kong, SAR 999077, People's Republic of China. ${ }^{5}$ Institute of Chinese Medicine and State Key Laboratory of Phytochemistry and Plant Resources in West China, the Chinese University of Hong Kong, Shatin, N.T, Hong Kong, SAR 999077, People's Republic of China. 'HKU-Pasteur Research Pole, School of Public
Health, Li Ka Shing Faculty of Medicine, The University of Hong Kong, Pok Fu Lam, Hong Kong, SAR 999077, People's Republic of China. ' Li Dak Sum Yip Yio Chin R \& D Centre for Chinese Medicine, The Chinese University of Hong Kong, Shatin, N.T, Hong Kong, SAR 999077, P. R. China.

\section{Received: 2 February 2018 Accepted: 22 April 2018}

Published online: 08 May 2018

\section{References}

1. Regoes RR, Bonhoeffer S. Emergence of drug-resistant influenza virus: population dynamical considerations. Science. 2006;312(5772):389-91.

2. Klenk HD, Garten W, Matrosovich M. Molecular mechanisms of interspecies transmission and pathogenicity of influenza viruses: lessons from the 2009 pandemic. BioEssays. 2011;33(3):180-8.

3. Yang S, Chen Y, Cui D, Yao H, Lou J, Huo Z, Xie G, Yu F, Zheng S, Yang Y, et al. Avian-origin influenza a(H7N9) infection in influenza a(H7N9)-affected areas of China: a serological study. J Infect Dis. 2014;209(2):265-9.

4. Kamali A, Holodniy M. Influenza treatment and prophylaxis with neuraminidase inhibitors: a review. Infect Drug Resist. 2013;6:187-98.

5. Furuta Y, Takahashi K, Kuno-Maekawa M, Sangawa H, Uehara S, Kozaki K, Nomura N, Egawa H, Shiraki K. Mechanism of action of T-705 against influenza virus. Antimicrob Agents Chemother. 2005;49(3):981-6.

6. Vanderlinden E, Vrancken B, Van Houdt J, Rajwanshi VK, Gillemot S, Andrei G, Lemey P, Naesens L. Distinct effects of T-705 (Favipiravir) and ribavirin on influenza virus replication and viral RNA synthesis. Antimicrob Agents Chemother. 2016;60(11):6679-91.

7. Bai GR, Chittaganpitch M, Kanai Y, Li YG, Auwanit W, Ikuta K, Sawanpanyalert P. Amantadine- and oseltamivir-resistant variants of influenza a viruses in Thailand. Biochem Biophys Res Commun. 2009;390(3):897-901.

8. Newman DJ, Cragg GM. Natural products as sources of new drugs over the 30 years from 1981 to 2010. J Nat Prod. 2012;75(3):311-35.

9. Koh A. Wen Bing (warm diseases) and the $2009 \mathrm{H} 1 \mathrm{~N} 1$ influenza. Australian journal of acupuncture and. Chin Med. 2010;5(2):23-9.

10. Liu Y, Ahmed S, Long C. Ethnobotanical survey of cooling herbal drinks from southern China. J Ethnobiol Ethnomed. 2013;9:82

11. Wu QF, Zhu WR, Yan YL, Zhang XX, Jiang YQ, Zhang FL. Anti-H1N1 influenza effects and its possible mechanism of Huanglian Xiangru decoction. J Ethnopharmacol. 2016;185:282-8.

12. Peng $X Q$, Zhou HF, Zhang $Y Y$, Yang $J H$, Wan HT, He $Y$. Antiviral effects of Yinhuapinggan granule against influenza virus infection in the ICR mice model. J Nat Med. 2016;70(1):75-88.

13. Rong R, Li RR, Hou YB, Li J, Ding JX, Zhang CB, Yang Y. Mahuang-Xixin-Fuzi decoction reduces the infection of influenza a virus in kidney-Yang deficiency syndrome mice. J Ethnopharmacol. 2016;192:217-24.

14. Ding Y, Zeng L, Li R, Chen Q, Zhou B, Chen Q, Cheng PL, Yutao W, Zheng J, Yang $Z$, et al. The Chinese prescription lianhuaqingwen capsule exerts antiinfluenza activity through the inhibition of viral propagation and impacts immune function. BMC Complement Altern Med. 2017;17(1):130.

15. Chen H, Jie C, Tang LP, Meng H, Li XB, Li YB, Chen LX, Yan C, Kurihara H, Li $Y F$, et al. New insights into the effects and mechanism of a classic traditional Chinese medicinal formula on influenza prevention. Phytomedicine. 2017;27:52-62.

16. Kao RY, Yang D, Lau LS, Tsui WH, Hu L, Dai J, Chan MP, Chan CM, Wang P, Zheng $B J$, et al. Identification of influenza a nucleoprotein as an antiviral target. Nat Biotechnol. 2010;28(6):600-5.

17. Gaush CR, Smith TF. Replication and plaque assay of influenza virus in an established line of canine kidney cells. Appl Microbiol. 1968;16(4):588-94.

18. Liu G, Xiong S, Xiang YF, Guo CW, Ge F, Yang CR, Zhang YJ, Wang YF, Kitazato K. Antiviral activity and possible mechanisms of action of pentagalloylglucose (PGG) against influenza a virus. Arch Virol. 2011;156(8): 1359-69.

19. Bardsley-Elliot A, Noble S. Oseltamivir. Drugs. 1999;58(5):851-60. discussion 861-852

20. Vervelde L, Reemers SS, van Haarlem DA, Post J, Claassen E, Rebel JM, Jansen CA. Chicken dendritic cells are susceptible to highly pathogenic avian influenza viruses which induce strong cytokine responses. Dev Comp Immunol. 2013;39(3):198-206.

21. Loo YM, Gale M Jr. Influenza: fatal immunity and the 1918 virus. Nature. 2007;445(7125):267-8. 